\title{
Les médiamorphoses de Chloé Delaume
}

\author{
Alexandra Profizi \\ Université Sorbonne Nouvelle-Paris 3 \\ France
}

\section{Introduction}

Se décrivant comme «personnage sans domicile fixe »1, Chloé Delaume a vécu dans divers espaces. Elle a quelques fois déménagé au cours de sa vie humaine, mais surtout au cours de son œuvre, à travers le pays de l'Autofiction. Elle a parfois emmené ses chats, et toujours de lourds bagages puisqu'elle ne se déplace pas seule: Chloé Delaume, c'est une narratrice, un personnage, une autrice - trois entités, au moins, pour un unique nom. Sous cette triple étiquette s'exprime une puissante voix narrative. Cependant, elle ne se suffit pas d'une voix, puisque la corporalité est, dans son œuvre, essentielle. Cette voix cherche inlassablement à s'incarner dans une chair, qu'elle soit physique, de papier ou virtuelle. Sa pratique ludique des différents médias (du livre au théâtre en passant par les réseaux sociaux ou le jeu vidéo), lui permettant notamment de mettre en lumière la malléabilité du personnage de fiction, est l'une des caractéristiques centrales de son œuvre, et en fait l'une des pionnières de la littérature transmédiatique en France.

Chloé Delaume subit, ou s'impose, de nombreuses métamorphoses, c'est-à-dire des transformations profondes de son être; plus précisément des changements de forme par le biais de l'utilisation de différents médias, que nous proposons d'étudier succinctement ici. L'exploration de la déstructuration identitaire est toujours accompagnée d'une sorte de déstructuration, voire de destruction, formelle qui lui fait écho. L'hybridité - notion capitale dans l'œuvre delaumienne - se retrouve à deux niveaux : au niveau diégétique (car elle manipule la frontière entre le réel et la fiction), et au niveau médiatique (car elle manie et mêle souvent plusieurs supports). Très tôt, Delaume a pris en compte les nouvelles technologies dans son travail, dans un geste double, nous semble-t-il : d'une part, comme une main tendue à la pop culture, et, d'autre part, comme pratique expérimentale autour de la distorsion des limites du roman. Régis Debray affirme que la pensée s'articule autour du média par lequel elle s'exprime. D'après la discipline qu'il a nommée la « médiologie ", l'étude d'une pensée est indissociable de l'étude du support qui la véhicule. C'est pourquoi il nous semble nécessaire de nous pencher sur le phénomène de médiamorphoses mis en œuvre par Chloé Delaume. Notons que, s'il existe deux acceptions principales du terme «médiamorphose » (conceptualisées par Roger Fidler en 1997, et Alfred Smudits en 2002), elles renvoient toutes deux aux diverses mutations des médias à travers le temps. Ici, au contraire, nous utilisons ce terme en référence aux mutations de Chloé Delaume en fonction des médias qu'elle utilise.

L'écriture de Delaume parcourt les possibilités médiatiques de son époque. Toutefois, au-delà de l'expérimentation formelle, voire psychologique dans certains cas, comment la parole se développe-t-elle à travers cette démultiplication de supports autour du récit de soi? Comment le travail sur l'identité se traduit-t-il au sein des outils utilisés par Chloé Delaume? Nous allons voir comment le phénomène de médiamorphoses qui prend place dans son œuvre peut nous éclairer sur son programme littéraire, et plus précisément sur son projet de réappropriation identitaire.

\section{Fiction et diffraction}

Pour sonder le mécanisme de médiamorphoses, il s'agit avant toute chose de se pencher sur le personnage dans les œuvres de Chloé Delaume. En effet, elle construit une identité déclinée, entre « l'auteur, la narratrice et l'héroïne », cette « trinité » qui compose l'autofiction. Elle revendique la multiplicité (« Elle + elle, elles + elles, toutes : c'est moi » (Une femme avec personne dedans 128), dans le but de se consolider :

Je m'appelle Chloé Delaume. Je suis un personnage de fiction. Livre et vie s'entremêlent, mon Moi en trois parcelles2, auteur, narratrice, héroïne. Je suis d'une trinité forcée de s'incarner, sous peine d'être expulsée par n'importe quel autrui. À cet instant j'affirme : j'écris ce que j'ai vu, ce qui est, ce qui doit arriver ensuite. J'écris et je m'écris, car je suis l'héroïne. Ainsi sera le pacte qui me lie avec l'ange tout autant qu'avec vous. (Une femme 16)

L'affirmation « Je m'appelle Chloé Delaume, je suis un personnage de fiction », répétée dans chacune de ses 
œuvres, est centrale dans son écriture. Elle engendre la première et la plus importante métamorphose, dans un geste performatif. D'une part, c'est à partir de celle-ci que s'enclenche le processus de fictionnalisation de l'écrivain, par une assimilation entre le narrateur-personnage et l'être de chair et d'os dont le nom figure sur la couverture. D'autre part, c'est aussi le point de départ de la reconstitution de soi par soi-même : face aux fictions collectives qui dépossèdent l'individu de son vécu, l'écriture permet au contraire de s'approprier l'identité souhaitée. Nous ne nous étendrons pas davantage sur ces thématiques bien connues de l'œuvre delaumienne, largement et brillamment traitées par ailleurs (Cornelio 2017), et par l'autrice elle-même (« S'écrire mode d'emploi »).

Elle compare l'opération de fictionnalisation par l'autofiction à un phénomène magique, pour lequel il est nécessaire de détenir les formules et le mode d'emploi (" Pour être un personnage de fiction dans la vie et une héroïne dans ses textes, il faut le vrai grimoire avec le bon rituel. " (Une femme 13). Elle nomme ainsi « transmutation » 3 (Une femme 13) cette conversion de soi, de la réalité à la fiction, avec pour but la modification du réel. Sa conception théorique de l'autofiction, ainsi que sa démarche dans la constitution d'une identité fictionnelle et la création d'une instance auto-narrative proches du rituel magique de transformation sont les motifs du dispositif de médiamorphose.

Parallèlement à l'acte d'autocréation par la parole, Chloé Delaume cherche à prendre différentes enveloppes corporelles, textuelles et médiatiques, présentées comme autant d'hôtes successifs. Elle investit, par exemple, l'univers de la série télévisée Buffy contre les vampires dans La nuit je suis Buffy Summers, un livre dont vous êtes le héros. Elle fait une expérimentation sur "le temps de cerveau disponible » dans J'habite dans la télévision. Elle pénètre le jeu vidéo Les Sims, dans Corpus Simsi. Et encore, elle publie une «fiction numérique », un livre sous forme d'application, uniquement digital, Alienare, sans oublier ses œuvres destinées au théâtre, à la radio et à la télévision (Au commencement était l'adverbe, Transhumances, La Contribution, pour ne citer que ceux-là).

\section{Un corps contre un support}

Le statut du corps, tel qu'il s'exprime dans Une femme avec personne dedans, est d'abord paradoxal car, si la corporalité est nécessaire pour ancrer la présence du personnage dans la fiction, il faut aussi pouvoir s'en évader. Dans ce roman, tout d'abord, le corps a été « déserté » (Une femme 15) : il est présenté comme une simple enveloppe, un « corps reflet apparence » (Une femme 17). Delaume esquisse une description physique rapide mais sévère de son personnage : « Il est temps à présent d'observer l'héroïne. Silhouette plus qu'alourdie depuis le dernier épisode, une quinzaine de kilos. Cheveux noirs, pupille fixe. Gros plan. Epiderme tendu, comédons mais absence de rides. Est-elle jolie, je n'en sais rien, d'ailleurs ça n'a pas d'importance. " (Une femme 17) Nous retrouvons dans cet extrait la description fragmentée du corps, analysée par Dawn Cornelio (« De Nathalie Dalain à Chloé Delaume : qui est qui? ») comme l'un des traits récurrents dans l'œuvre de Delaume, signalant la mise en morceau de soi. Or, ici, la description de l'apparence du personnage, topos du roman réaliste, est rapidement évacuée, avant d'être carrément discréditée et rendue inutile, car « la chair même se dissout dans la littérature. Et cela dès son contact. La pulpe qui s'enfonce dans les touches du clavier, une volupté superficielle. Ce qui est, ce n'est plus qu'elle; lancement du générique. » (Une femme 17) L'identité fictionnelle que l'autrice établit pour sa personne n'a donc plus besoin de corps, puisqu'elle existe dans le roman. Son corps représente une étendue lisse, le personnage est un réceptacle, " une surface à transfert 》 (Une femme 75) laissée libre à l'investissement. Après avoir quitté son corps réel, même l'identité fictive que Chloé Delaume a créée se vide de l'intérieur; elle rêve de « nénuphars 4 fanés dégorge[ant] un jus rance tout autour de son lit » (Une femme 103), qui en se vidant peuvent illustrer le sentiment d'expulsion. Progressivement, dans le roman, son enveloppe et son « Je » se vident, ne laissant plus qu'une « femme avec personne dedans ».

Double de l'autrice, le personnage sert avant tout de support au langage, par lequel il est traversé. Sa fonction est caractérisée par sa langue : "II se peut par ailleurs que je ne sois qu'un médium, un canal aux parois parfaitement récurées. [...] J'effectue une sortie, je ne suis plus dans mon corps. Quiconque peut s'introduire et en prendre les commandes. Quiconque possède ce petit livre " (Une femme 107). Ici, le « quiconque » évoqué renvoie au lecteur, quels que soient son parcours ou ses intentions, passant de récepteur à protagoniste. L'autrice semble ne plus avoir d'autre intention que de nous laisser accéder au corps de son personnage tel un instrument mis à disponibilité. Cependant, dans l'un des chapitres finaux, elle fait un revirement total, elle résiste et réaffirme son pouvoir face au processus d'identification. Elle déclare ainsi au lecteur qui entendait effectuer un transfert : 
Je vais vous décevoir : vous ne pourrez en rien agir sur ce récit, à peine la porte franchie vous voilà menotté. Ce n'était qu'un appât, le piège s'est refermé, l'acier mord votre gorge, vous êtes à ma merci. [...] Vous êtes seul avec moi, poignets liés au chapitre une consentante victime, taisez-vous, écoutez. (Une femme 115)

En réalité, la transparence et la mise à disposition de soi n'étaient qu'apparences. Il s'agissait d'un leurre, d'une amorce pour faire entrer le lecteur dans la fiction. Une fois la lecture débutée et le lecteur accroché (le lexique de la pêche et de l'incarcération montre la violence intentionnelle du procédé), l'autrice reprend pleinement le contrôle de l'histoire et de la parole qui surgissent.

\section{De corps en corps : les métalepses médiatiques}

L'avatar, double virtuel - Parmi les différents corps expérimentés par Chloé Delaume, l'un des plus intéressants est certainement le Sims. Dans Corpus Simsi, elle passe en effet à l'étape supérieure dans l'entreprise de médiamorphoses en se déplaçant dans le monde virtuel. Jusqu'ici, le personnage Chloé Delaume se déplaçait de texte en texte, de fiction en fiction. Avec le projet Corpus Simsi, entamé sur un blog avant de devenir un roman publié, elle quitte son corps humain et traverse l'écran. Au-delà du métadiscours qui parcourt toute son œuvre et fonde la construction performative de son identité en tant que personnage, ce roman semble être lui-même une illustration de la métalepse, dans sa structure. Si la métalepse désigne l'intrusion d'un niveau narratif dans un autre, nous voyons ici le redoublement du procédé métaleptique, avec l'intrusion d'un médium, le jeu vidéo, dans un autre médium, le livre, par le biais de la personne-personnage-avatar Chloé Delaume, ainsi que des multiples captures d'écran qui peuplent les pages du roman. Elle y crée un avatar à son image, selon le principe de ce jeu vidéo5. Dans le premier chapitre, intitulé « Configuration du personnage », on la voit choisir l'apparence physique et les traits de caractère de son Sims. À nouveau, l'avatar devient le support de la fiction, avec toutes les possibilités et contraintes que cela engendre. De plus, nous pouvons remarquer que le corps réel, apparemment abandonné, n'a pourtant jamais été aussi présent au sein d'un livre : son image virtuelle peuple l'ouvrage grâce aux captures d'écran qui le parcourent. À travers des captures d'écran de parties, l'avatar de Chloé Delaume se promène de page en page, changeant de costume mais toujours présenté sous les traits
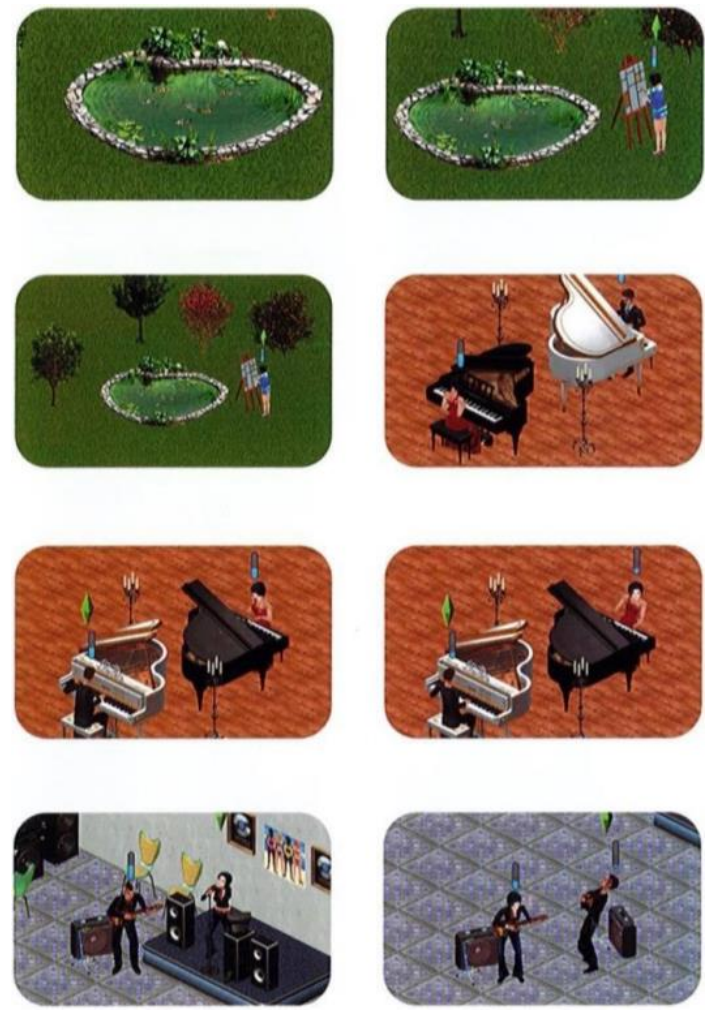
de l'autrice que l'on connaît. L'effet d'hybridité entre le corps réel et son avatar virtuel était d'ailleurs d'autant plus fort au moment de la parution du livre en 2003, puisque celle-ci a été précédée de nombreuses performances de l'autrice, sur scène, son corps se superposant ainsi aux projections du jeu vidéo sur grand écran.

À propos de son avatar, le personnage déclare : « me voici désormais à l'image du corps qui me refoula. Me voici à l'image du corps qui me refusa. " (Corpus Simsi 14). Expliquant cette volonté de créer un avatar à l'image de son corps réel, Jean-Claude Heudin écrit dans Robots et Avatars : " la majorité des visiteurs des mondes virtuels préfèrent être représentés par des clones virtuels à leur image. Ils ont besoin d'une libération du corps pour conquérir de nouveaux espaces » (123). C'est bien le projet auquel s'attèle Chloé Delaume qui, débarrassée des contraintes du corps physique par la médiamorphose, va se servir de l'espace virtuel pour approfondir ses réflexions récurrentes, c'est-à-dire disloquer la frontière entre le réel et la fiction, et surtout pousser son travail de réappropriation identitaire à son paroxysme, jusqu'à la crise (existentielle, théorique et formelle, comme nous le verrons ci-dessous).

Corpus Simsi 81. Reproduite avec la permission de l'autrice. 
Incarnation et conséquences - Le personnage de fiction Chloé Delaume a donc quitté, en apparence, le monde des livres. II prend son indépendance par rapport à l'autrice. II y a un renversement du pouvoir : exclu et coincé derrière son écran, dans "le monde réel trop organique ", l'autrice déchue ne peut plus agir sur son narrateurpersonnage de pixels qui entend s'ancrer dans le virtuel et s' « encrer » (Corpus Simsi 114) dans la fiction, hors d'atteinte. Seul, ce dernier parvient à s'élever en figure christique réincarnée, ressuscitée, " Corpus Simsi ». L'idée de la réincarnation christique est renforcée par l'étymologie du terme " avatar », qui provient du sanskrit " avatâra », désignant notamment l'incarnation corporelle d'une entité supérieure dans le monde des mortels pour accomplir une tâche précise. Dans I'hindouisme, il désigne les incarnations de Vishnou. Ici, au lieu d'une divinité qui s'incarne dans un corps matériel, c'est un corps matériel qui se désincarne dans une représentation virtuelle.

Cependant, la réincarnation est-elle totale? Le sous-titre indique son caractère réversible : « Incarnation virtuellement temporaire ». D'une part, sa durée est limitée (" temporaire »), d'autre part, elle est seulement "virtuelle », soumise au jeu vidéo. Selon le narrateur, le virtuel rend plus réel et les Sims sont donc, en fait, encore plus humains que les Hommes eux-mêmes: "Simulation de vie virtuelle, nous vivons plus, bien plus que vous. Vous qui êtes du réel, vous qui êtes palpables jusqu'aux caries dentaires, tangibles jusqu'au varices. » (Corpus Simsi 28). Encore une fois, ce qui compte avant toute chose, c'est le langage : « les personnages de fiction sont faits de verbe. ", rappelle Delaume. Avec ironie, le personnage de fiction qui semblait avoir trouvé l'environnement idéal dans le virtuel se retrouve finalement dans la même situation que le lecteur face au livre, c'est-à-dire dans l'impossibilité de discerner les différentes couches de cet enchevêtrement diégétique. Le narrateur comme le lecteur font face à la dégénérescence de cet univers. L'instance narrative, mettant en évidence l'impossibilité d'identifier son appartenance à un univers diégétique, entre le réel, le fictionnel et le virtuel, se présente comme trouble et instable. Son statut se trouve en conformité avec le projet revendiqué de remise en cause des mécanismes de la fiction et de la réalité.

Ainsi, dans le cadre de Corpus Simsi, l'affirmation-incantation « Je m'appelle Chloé Delaume, je suis un personnage de fiction ", ici répétée de nouveau, devient particulièrement problématique dans la tentative d'identifier d'où s'exprime l'énonciateur. En effet, si Chloé Delaume est un personnage de fiction, alors comment envisager son Sims qui prend la parole, et qui est lui-même personnage de jeu vidéo? II s'agirait donc du personnage de fiction d'un personnage de fiction? Finalement, cette instance narrative hybride ne s'ancre ni dans un être de papier, ni dans un être de pixels. S'il porte toujours le même nom, livre après livre, le narrateurpersonnage chez Chloé Delaume se définit comme un démultiplicateur de vies, qui permet d'entreprendre une négociation permanente avec le champ des possibles : "Vous savez que ce livre est composé de réel autant que de fiction. Au milieu est ma vie. » (Une femme 107). Delaume dans Corpus Simsi s'est détournée du réel, pour explorer les promesses du monde parallèle qu'est le virtuel. Pourtant, en menant à bien cette entreprise, elle passe par l'objet livre, mettant à jour un jeu ironique puissant. Elle se pose non seulement en écrivain qui cherche comment illustrer le rapport conflictuel entre réel et fiction, mais également elle-même, en tant qu'individu qui cherche à reconstruire son identité, entre réel et virtuel, se mettant en situation de difficulté. Elle parvient à dénoncer, par la médiamorphose, les impasses de la fiction. Ici, son écriture atteint le paroxysme du dédoublement, de l'éclatement de l'identité narrative, pour montrer comment opère la distance de soi à soi.

\section{Environnement numérique et dédoublement}

Après avoir vu ce cycle dans le monde virtuel et avant de poursuivre avec le numérique, il s'agit néanmoins de préciser que, malgré la quantité de médiamorphoses effectuées par Chloé Delaume, il en demeure une à laquelle elle résiste, alors que pourtant nous nous y livrons tous, ou presque : il s'agit des réseaux sociaux, et en particulier de Facebook. En effet, tandis que les réseaux sociaux peuvent représenter le lieu privilégié de la médiamorphose, puisque l'on se doit d'y créer un double de nous-mêmes pour exister, l'autrice semble ne pas parvenir à laisser libre cours à Chloé Delaume personnage de fiction. Elle dispose bien d'un compte Facebook qu'elle alimente, mais elle a attribué sa page à son chat, Miss Tick6, présentée comme l'administratrice du compte au nom de Chloé Delaume, comme l'indique la biographie : «Interface gérée par Miss Tick, siamoise et domestique qui se tape tout le sale boulot ». Sur les publications, c'est le chat qui s'exprime directement à la première personne (par exemple, cette publication du 20 mars 2017 : « Miss Tick prévient : "Contrairement à ce qu'elle s'obstine à croire, ma maîtresse est physiquement peu résistante, et j'ai beau être une siamoise super futée, je ne peux pas répondre à ses mails. C'est donc avec désarroi que je vous annonce la fermeture de la TPE Chloé Delaume pour quelques jours." »). Pourtant, les publications la concernent bien elle, écrivain - ce n'est pas un compte à l'effigie du chat. Delaume a donc créé une figure qui s'apparente plus au narrateur qu'au personnage : le chat n'est présent que pour partager des informations sur ce qui arrive à sa maîtresse, qui reste 
le personnage central de l'histoire. De plus, elle opère une mise à distance ironique, en utilisant son chat; ce procédé s'inscrit dans la tradition de l'animal narrateur (notamment dans l'univers des contes), ainsi que dans l'imagerie de la sorcière qui lui est chère. La médiation de la prise de parole par l'animal montre la volonté de Delaume de mettre à distance ce qui est dit sur Facebook. Elle semble nécessiter un tiers pour s'exprimer sur cette plateforme, comme un moyen de dédramatiser ou de détourner la parole, toujours avec humour. Pour le dire rapidement, l'absence de médiamorphose sur Facebook, c'est-à-dire l'impossibilité d'y affirmer "Je m'appelle Chloé Delaume, je suis un personnage de fiction », pourrait s'expliquer par le fait que ce réseau social n'est pas perçu comme un support de fiction par l'autrice, cet espace semble relever du domaine de la communication et n'est pas considéré comme une ressource de création esthétique ni d'expression littéraire.

Le personnage Chloé Delaume se construit et s'affirme œuvre après œuvre autour de divers médias qui infléchissent sa perception et son discours. Ces expérimentations sur des formes médiatiques différentes sont aussi l'occasion de délocaliser - temporairement - le laboratoire de l'écrivain. Nous allons donc évaluer à présent l'impact de cette écriture médiamorphique en relation aux conceptions de l'autrice des notions de livre et d'œuvre.

\section{Aux frontières du texte}

Au-delà de l'invasion du virtuel opérée dans Corpus Simsi, Chloé Delaume s'est emparée très tôt de l'environnement numérique pour mener plus d'expérimentations sur le personnage ainsi que sur l'écriture. Ces pratiques créatives profondément collectives nées sur internet nous poussent à interroger les contours de l'œuvre de l'autrice. Où se placent les contenus numériques à l'égard de l'œuvre publiée? En effet, l'œuvre de Delaume sur internet s'approche dangereusement des frontières du texte, délogé du livre. II s'agit d'une démarche que l'on retrouve dans sa pratique de l'écriture sur internet, où elle complète ou prépare ses livres avec la publication d'archives et de travaux rendus accessibles aux lecteurs, comme en témoigne par exemple la section « Cycle en cours » de son site web. Elle exhibe la rédaction en cours sur le mode du work in progress. Sur son site, elle a ainsi créé une véritable base de données autour de son œuvre, qui semble indispensable pour appréhender cette dernière dans sa globalité. La plupart de ses livres étant perçus comme des cycles ou des expériences qui ne se limitent pas seulement au texte publié, la lecture de ses romans serait probablement amputée si elle n'était pas complétée par la consultation de publications sur le site de l'autrice. Celui-ci devient aussi un lieu recueillant l'écriture sur l'écriture : « II ne s'agit plus seulement d'une médiation du livre via le réseau, mais d'une présence tierce du livre, un livre à côté des livres. " (Gervais \& Saemmer). II semble y avoir un double discours - les commentaires et réflexions7 menés sur internet existent à côté de l'œuvre, elles la précèdent et la prolongent. L'écriture est dédoublée, entre deux supports et deux finalités, se trouvant néanmoins liées et nourrissant nécessairement l'œuvre.

La diffraction des paroles littéraires de l'autrice interroge la figure auctoriale telle que nous la connaissons : la possibilité d'exprimer toutes sortes de discours sur divers supports et sous divers registres, au fil des médiamorphoses, en fait une figure mouvante. À la fois puissante et instable, affirmant une présence forte et dévoilant ses hésitations, Chloé Delaume bénéficie d'un statut en rupture avec les schémas anciens. Occupant tous les espaces grâce à d'innombrables médiamorphoses, elle œuvre à l'émergence d'une auctorialité inédite.

\section{Contre une littérature figée : la médiamorphose comme issue}

D'autre part, depuis ses débuts, Chloé Delaume prend position contre une idée figée de la littérature et du livre. Selon elle, le livre ne doit pas se limiter à l'objet sous forme de pages de papier. La littérature dépasse son contenant traditionnel; elle peut inclure d'autres formes de technologies, accueillant la fiction tout aussi bien que le fait le roman. Avec La Nuit je suis Buffy Summers, elle nous met en garde contre la sacralisation du papier :

Le lecteur entretient un curieux fétichisme avec l'objet dit livre, attachement synecdoque, respect face au papier. À ceux qui ne cornent jamais, protègent les couvertures, il est dit faites un geste, apprenez à toucher. Premier point de contact, intervention physique. Vous comprenez enfin que ce n'est qu'un support, un support de fiction. Qui peut être malléable et soumis au trafic, à la circulation. (13)

Nombre de ses projets attestent ce souhait de décloisonnement : par exemple, son livre-application Alienare, évoqué plus haut, ou encore la collection qu'elle a fondée et dirigée chez Joca Seria, Extraction, regroupant 
texte, son et vidéo, dans le but affiché de se détacher de la nécessité du papier. L'idée du livre renvoie généralement au texte, qui se définit comme un discours clos, achevé. Nous avons vu que ce discours clos se trouve fissuré chez Delaume, les signes glissent d'un plan diégétique à un autre : de la personne au personnage - de la réalité à la fiction, ou inversement. L'idée du livre en tant que tout cohérent pose donc des limites, que Chloé Delaume dépasse. Son écriture diffractée par les médiamorphoses perturbe et résiste à la construction du texte; elle reste rétive à la forme imposée par l'idée du livre.

Ainsi, tandis que le papier fige, internet se présente comme la promesse d'une liberté de publication, de modification ou de précision. Cependant, ce type d'écriture mêlant plusieurs supports autour d'une œuvre présente un risque dans la mesure où certains éléments contemporains de la publication du livre ne sont plus accessibles. Le problème se pose déjà pour certaines de ses œuvres : par exemple, le blog lié au cycle de Corpus Simsi, celui lié à J'habite dans la télévision, ou encore la bande-son qui accompagne Dans ma maison sous terre ne sont plus disponibles sur internet. L'indisponibilité ou la disparition de certains documents est modérée par l'apparition de l'outil « Wayback Machine », une interface publique permettant d'accéder à des archives de sites web par le biais de captures instantanées de millions de pages. Leur usage dans le cadre des documents accompagnant certains livres de Chloé Delaume reste toutefois relatif : le fait de devoir aller les chercher sur des archives en limite l'accès. La démarche n'est plus la même, il ne s'agit plus d'éléments rendus disponibles par l'autrice elle-même, poussant à une lecture conjointe. De plus, si un nouveau lecteur ne sait pas que ces informations parallèles existent, il ne va pas les rechercher.

\section{Dialogisme circulaire}

Chloé Delaume déjoue donc sans cesse les attentes du lecteur car son personnage bondit de cadre en cadre, et de support en support, si bien qu'il semble rester uniquement une voix, qui se fait entendre depuis divers lieux. Son écriture bâtit ce que l'on pourrait qualifier de nouvel espace de dialogisme, pouvant se définir par sa circularité. Entre les œuvres littéraires mêlant divers supports, et l'expression sur le web, sont engendrés des doubles de soi. Plusieurs régimes discursifs sont rattachés à son identité, mais aucun ne suffirait, seul, à appréhender son projet littéraire dans sa totalité. Son énonciation se décline en plusieurs instances qui se croisent et se recoupent. Les médiamorphoses font donc apparaître une forme de dialogisme interne circulaire, pouvant être défini comme l'interaction des discours de différentes entités renvoyant à l'autrice, sur le principe d'une polyphonie propre à l'autrice elle-même: son expression prend plusieurs voix, sur plusieurs supports, répondant à différents régimes auctoriaux, et partageant une continuité les unes avec les autres. Elle ne se pose pas seulement dans une représentation de l'écrivain interne au texte, ni dans une position totalement externe, ni même seulement comme personnage de fiction, mais comme un assemblage de ces figures permises par son utilisation privilégiée de la médiamorphose et par l'hybridité du récit de soi déployé sur une multiplicité de supports. Son expression se propage dans différents espaces qu'il est nécessaire de distinguer, mais qui semblent indissociables.

\section{Conclusion}

Chloé Delaume élabore une nouvelle forme d'auctorialité à partir de variations de son personnage opérées lors de ses métamorphoses. Elle se pose en «personnage de fiction pire que les autres » car il a refusé de s'incarner dans un livre. Dans chacun de ses romans, elle tente de prendre possession d'un lieu de résidence, mais ce sentiment n'est toujours que temporaire. A chacune des médiamorphoses, la corporalité du personnage se révèle incertaine pour finalement mieux reprendre pleine possession de son autorité. Son œuvre représente un éclatement des « moi » et de la parole de l'autrice.

Si l'autofiction est l'espace de l'entre-deux, Chloé Delaume fait totalement sienne cette propriété et en fait même la terre de l'entre-mille : elle exploite jusqu'au vertige les ressources de la fiction de soi par le biais de médiamorphoses. Alliant les mécanismes diégétiques aux outils formels voire techniques (technologiques) qui lui sont offerts, elle tente de résoudre, par-là, les trajectoires théoriques parfois contradictoires que ce genre fait surgir ou que son œuvre et son travail de reformation identitaire mettent au jour.

Pourtant, il est nécessaire de noter que sa démarche évolue dans le temps, le recours à la médiamorphose n'est pas constant dans son œuvre. Ce recours peut s'arrêter, ou être mis entre parenthèses, comme en témoigne la publication en 2016 des Sorcières de la République, roman non autofictionnel et au format papier traditionnel (même si le projet est né d'expériences menées dans le réel, et documentées sur son site). Inversement, elle 
effectue finalement un retour sur Facebook et reprend pleine possession de l'expression sur cette plateforme (« J'ai donc viré Miss Tick et le mode veille ici. Je vais juste prendre FB comme mon vieux blog sauf que cette fois la vitrine sera ouverte. Mais là le dispositif, c'est un forum en pire, donc je dois vraiment faire des travaux. ", déclare-t-elle le 4 juin 2018 sur son compte Facebook).

Ainsi, ce principe d'écriture en mouvance permanente lui permet de résister à tout ancrage. Seule l'autrice détient encore le pouvoir. Chez Chloé Delaume, le texte n'est jamais clos et l'identité ne peut être figée. Rappelons pour finir les mots de Clotilde à Charlie Orphan, ce personnage qui tenta une révolte : "Vous n'êtes pas autonome, relié à mon clavier, il n'y a que moi pour décider» (Au commencement était l'adverbe 51).

\section{Notes}

${ }_{1}$ Article «Corpus Simsi » sur le site de Chloé Delaume, http://www.chloedelaume.net/?page_id=294.

2 Je souligne le terme «parcelles », afin d'attirer l'attention sur le vocabulaire topographique souvent utilisé par l'autrice en référence à son être, comme espace à conquérir, parcourir ou cultiver.

3 La transmutation renvoie au changement d'une chose en une autre de façon générale, mais le terme contient également l'idée de magie, puisqu'il définit la transformation des métaux par les procédés de l'alchimie.

4 Le nénuphar est l'un des symboles du personnage Chloé Delaume. II s'agit, bien sûr, d'une référence à la maladie de la Chloé de Boris Vian, de qui elle tient son prénom.

5 Jeu vidéo de simulation de vie lancé en 2000, dans lequel le joueur doit, par le biais d'un avatar doté d'un physique humain et d'une « personnalité » qu'il lui attribue, maintenir un logement, un emploi et des relations sociales. La règle du jeu est ainsi résumée par Chloé Delaume : « Construire Acheter et Vivre ».

6 Notons également qu'elle avait d'abord tenté de se mettre à Facebook avec un profil au nom de son personnage Clotilde Mélisse en 2011, mais celui-ci a duré moins d'un an, et servait principalement à la préparation du projet «Extraction ».

7 Voir, par exemple, les évolutions du manuscrit de Mes biens chères sœurs (Seuil, 2019), renseignées sous forme d'« épisodes » sur son site (au sein de l'onglet « Sororisation générale », http://www.chloedelaume.net/?cat=24). Chloé Delaume a publié des articles au cours de la conception puis de la rédaction du texte. Les lecteurs assidus ont ainsi pu voir, en temps réel, les transformations et changements de direction opérés par l'autrice (à commencer par le titre, le texte étant initialement intitulé Sororisation générale puis Aujourd'hui Mesdames), jusqu'au moment de la publication du livre final.

\section{Bibliographie}

Cornelio, Dawn. «De Nathalie Dalain à Chloé Delaume : qui est qui? » S'écrire Chloé Delaume. Komodo 21, 2017. http://komodo21.fr/de-nathalie-dalain-a-chloe-delaume/

—. «Fragmentation des corps et des identités chez Chloé Delaume. »@nalyses. Revue de critique et de théorie littéraire, 11.1 (hiver 2016) : 135-159.

Delaume, Chloé. Corpus Simsi. Léo Scheer, 2003.

—. J'habite dans la télévision. Gallimard, 2006.

—. La Nuit je suis Buffy Summers. Éditions è®e, 2007.

—. Transhumances. Éditions è®e, 2007. 
—. «S'écrire mode d'emploi ». Colloque de Cerisy sur l'autofiction, publie.net, 2008.

—. Dans ma maison sous terre. Seuil, 2009.

—. Au commencement était l'adverbe. Joca Seria, 2010.

—. Une femme avec personne dedans. Seuil, 2012.

-. Alienare. Seuil, 2015.

—. Les sorcières de la République. Seuil, 2016.

—. La Contribution. Court métrage réalisé dans le cadre de la Collection «ÉCRIRE POUR » de Canal+, 2014.

—. Site web, http://chloedelaume.net/.

Findler, Roger F. Mediamorphosis: Understanding New Media. Pine Forge Press, 1997.

Gervais, Bertrand, et Alexandra Saemmer (dir.). « Esthétiques numériques, textes, structures, figures. 》 Protée, vol. $39, n^{\circ} 1$, printemps 2011 , p. 9-22.

Heudin, Jean-Claude. Robots et Avatars. Odile Jacob, 2009.

Jouve, Vincent. L'effet-personnage dans le roman. PUF, 1998.

Piva, Marika. «Formes kaléidoscopiques : I'hybridité chez Chloé Delaume », Babel, 33, 2016, pp. 139-159.

Smudits, Alfred. Mediamorphosen des Kulturschaffens (Musik und Gesellschaft). Braumüller, 2002.

Todorov, Tzvetan. Mikhaïl Bakhtine. Le principe dialogique. Seuil, 1981. 